\title{
Die Ermittlung von Ribonucleinsäure im Pflanzenmaterial
}

\author{
Von Hans-Georg Fritz und Bernd Röttger \\ Aus dem Max-Planck-Institut für Virusforschung, Tübingen \\ (Z. Naturforschg. 18 b, 124-132 [1963]; eingegangen am 17. August 1962)
}

\begin{abstract}
Zur Ermittlung der RNS in Pflanzenmaterial wurde die Methode von $\mathrm{Schmidt}-\mathrm{Th}$ a n $\mathrm{n}$ h a u s e r modifiziert, so daß nicht nur die Gesamtmenge an RNS, sondern auch deren Basenzusammensetzung bestimmt werden. Nach Entfernung der säurelöslichen Phosphate, der Lipoide und eines Teils der Farbstoffe wird die RNS in alkalischer Lösung zu Mononucleotiden hydrolysiert und diese durch Säulenchromatographie getrennt. Die Chromatographie wurde zu einer RoutineMethode ausgearbeitet, die ohne kostspielige Apparaturen und mit geringen Mengen an Elutionsmittel durchführbar ist. In verschiedenen Versuchsreihen wurde die optimale Hydrolysezeit mit 1-n. KOH bei $37^{\circ} \mathrm{C}$ zu $22 \mathrm{Stdn}$. ermittelt. Unter diesen Bedingungen wird nicht nur CMP, sondern auch AMP und UMP teilweise zerstört. Die infolgedessen notwendigen Korrekturfaktoren wurden ermittelt.
\end{abstract}

Die große Bedeutung der Nucleinsäuren für die Genetik und die Steuerung der Wachstums- und Vermehrungsprozesse erfordert die Ausarbeitung genauer Bestimmungsmethoden. Trotz zahlreicher Untersuchungen, eine der neuesten Zusammenfassungen ${ }^{1}$ umfaßt mehr als 400 Zitate, sind die bisherigen Methoden noch nicht in jedem Fall befriedigend. Dies gilt besonders für die Ermittlung der Nucleinsäuren im Pflanzenmaterial, die deshalb bisher wenig untersucht worden sind. Die Schwierigkeiten sind hier besonders groß, da infolge des Reichtums an Kohlenhydraten die üblichen kolorimetrischen Bestimmungen, die auf Reaktionen des Zuckeranteils der Nucleinsäuren beruhen, unsicher werden. Ferner enthalten die Pflanzen eine große Menge stark im UV-Bereich absorbierender Substanzen, so daß auch die spektrophotometrische Bestimmung der Nucleinsäuren gestört wird.

Das Ziel der vorliegenden Arbeit bestand darin, eine möglichst genaue und zuverlässige Methode für die Bestimmung der Ribonucleinsäure in Pflanzen auszuarbeiten. Sie sollte dabei so einfach sein, daß sie in jedem normal ausgestatteten biologischen Laboratorium durchgeführt werden kann und auch zur Serienbestimmung an zahlreichen Proben brauchbar ist. Neben der Absolutbestimmung der Ribonucleinsäure (RNS) sollte gleichzeitig das Molverhältnis der vier Mononucleotide erfaßt werden. Da Aussagen über den RNS-Gehalt, bezogen auf Frisch-

1 W. C. Hutchison u. H. N. Munro, The Analyst 86, 768 [1961].

2 W. C. Schneider, J. biol. Chemistry 161, 293 [1945].

3 M. Ogur u. G. Rosen, Arch. Biochem. Biophysics 25, 262 [1950].

${ }^{4}$ H. Kers, Planta 55, 259 [1960]. bzw. Trockengewicht, nicht wirklich vergleichbar sind, sollte gleichzeitig eine Absolutbestimmung der Desoxyribonucleinsäure (DNS) möglich sein. Man kann annehmen, daß der DNS-Gehalt der Zellzahl annähernd proportional ist, so daß dieser als Bezugssystem für den RNS-Gehalt sinnvoll erscheint. Von den drei Standardmethoden zur Bestimmung von RNS in Gegenwart von DNS ist die nach SCHNEI$\mathrm{DER}^{2}$ in unserem Fall für Pflanzenmaterial ungeeignet, da bei diesem Verfahren RNS und DNS nicht getrennt, sondern nebeneinander kolorimetrisch bestimmt werden, was hier aus den oben genannten Gründen nicht möglich ist. Das Verfahren von OGURRosen $^{3}$ erwies sich ebenfalls für unsere Zwecke als unbrauchbar, da mit verdünnter $\mathrm{HClO}_{4}$ in der Kälte die RNS aus dem Blattmaterial nicht herausgelöst werden konnte, ohne daß die DNS ebenfalls angegriffen wurde. Dies steht in Übereinstimmung mit Beobachtungen anderer Autoren ${ }^{4-7}$. Andere Extraktionsverfahren, z. B. mit 10-proz. NaCl-Lösung oder mit Phenol ${ }^{1}$, waren für quantitative Untersuchungen ebenfalls nicht geeignet, so daß wir uns schließlich zu einer Modifikation der Methode von Schmidt-Thannhauser ${ }^{8}$ entschlossen. Kritisch ist bei diesem Verfahren die alkalische Hydrolyse des Pflanzenmaterials. Es mußte gezeigt werden, daß die RNS vollständig zu Mononucleotiden hydrolysiert werden kann, ohne daß die Säurefällbarkeit der DNS beeinträchtigt wird. Weiterhin war es notwen-

5 J. H. McLendon, Amer. J. Bot. 39, 275 [1952].

6 M. Holden, Biochem. J. 51, 433 [1952].

7 P. O. P. Ts'o u. C. S. SAto, Exp. Cell. Res. 17, 227 [1959].

8 G. Schmidt u. S. H. Thannhauser, J. biol. Chemistry 161, 83 [1945]. 
dig, die Wirkung von Alkali auf die Mononucleotide näher zu untersuchen, denn es ist bekannt, daß durch die Alkalihydrolyse die Cytidylsäure angegriffen wird ${ }^{9,10}$.

Für die Bestimmung der Mononucleotide selbst arbeiteten wir, in Anlehnung an bekannte Verfahren, eine einfache säulenchromatographische Trennung aus, da die Papierchromatographie wegen der hohen Blindwerte zu ungenau ist. Um die Brauchbarkeit der Methode zu prüfen, wurde Blattmaterial von verschiedenen Arten der Gattung Nicotiana und der Gattung Epilobium sowie eine Reihe anderer Testsubstanzen verwendet.

\section{Durchführung der Analyse}

Die anschließend beschriebene Methode ist geeignet für die Bestimmung von RNS aus pflanzlichem Material in einem Bereich zwischen 0,1-10 $\mu \mathrm{Mol} /$ Mononucleotid, d. h. eine Messung erfordert mindestens $0,2-0,5 \mathrm{~g}$ Frischgewicht normales Pflanzengewebe. Die Mindestmenge ist abhängig von der Art des Pflanzenmaterials, es ist günstig, einer Bestimmung möglichst etwas größere Mengen $(1-3 \mathrm{~g})$ zugrunde zu legen.

\section{a) Vorbereitung des Ausgangsmaterials}

Nach Entfernung der Mittelrippen werden frisch geerntete Blätter gewaschen, grob zerkleinert, in einem mit einer Kältemischung von Methanol-Trokkeneis vorgekühlten Mörser sehr schnell unter $\mathrm{Zu}$ gabe von Trockeneis eingefroren, zerrieben und lyophylisiert. Das so erhaltene grüne Trockenpulver (GTS) wird fein zermahlen, trocken verschlossen hält es sich bei kühler Lagerung mehrere Wochen. In Anlehnung an die allgemein angewendete Methode zur Extraktion der säurelöslichen Phosphate ${ }^{1,13}$ werden unter Eisbadkühlung $20 \mathrm{ml}$ eiskalte 0,5-n. $\mathrm{HClO}_{4} / \mathrm{g}$ GTS zugegeben und $10 \mathrm{Min}$. gerührt. Nach Zentrifugation (5 Min., $2800 \mathrm{U} / \mathrm{min}$, Kühlzentrifuge) wird das Sediment zweimal mit eiskaltem Wasser (je $20 \mathrm{ml} / \mathrm{g}$ GTS) gewaschen. Die Lipide und ein großer Teil der Farbstoffe werden zunächst mit kaltem 96-proz. Äthanol $(20 \mathrm{ml} / \mathrm{g}$ GTS) und anschließend 3-5-mal, bis der Über-

9 D. H. Marrian, V. L. Sticer, M. E. Balis u. G. B. Brown, J. biol. Chemistry 189, 533 [1951].

10 J. N. Davidson u. R. M. S. Smellie, Biochem. J. 52, 594 [1952]. stand klar bleibt, mit Äthanol - Äther [3: 1 (v/v), $20 \mathrm{ml} / \mathrm{g}$ GTS] bei $55-65^{\circ} \mathrm{C}$ je 3 Min. extrahiert. Danach wird das Sediment zweimal mit Äther $(20 \mathrm{ml} / \mathrm{g}$ GTS $)$ gewaschen und nach erneutem Abzentrifugieren im Exsikkator unter Wasserstrahlvakuum getrocknet.

Das weißliche Trockenpulver (WTS) wird fein zermörsert und ist, verschlossen aufbewahrt, in der Kühltruhe mehrere Monate haltbar. Bei stark anthocyanhaltigem Material lassen sich diese Farbstoffe durch die Vorbehandlung nur z. T. entfernen, sie beeinflussen jedoch den weiteren Analysengang nicht.

\section{b) Alkalihydrolyse}

$100 \mathrm{mg}$ Trockenpulver (WTS), entsprechend 0,7 bis $3,5 \mathrm{mg}$ RNS, werden in $10 \mathrm{ml} 1-n$. KOH bei $37^{\circ} \mathrm{C}$ unter ständigem Rühren $22 \mathrm{Stdn}$. in einem Meßkölbchen hydrolysiert. Die Reaktion wird durch Abkühlen des Hydrolysats auf $0{ }^{\circ} \mathrm{C}$ unterbrochen, und auch alle weiteren Schritte werden bei möglichst tiefer Temperatur, $0-4{ }^{\circ} \mathrm{C}$, ausgeführt. Nach $\mathrm{Zu}$ gabe eines Tropfens einer 0,1-proz. wäßrigen Phenolrotlösung als Indikator wird mit 70-proz. $\mathrm{HClO}_{4}$ angesäuert, bis der Farbumschlag eintritt (etwa 0,8 bis $0,9 \mathrm{ml} / 10 \mathrm{ml}$ ). Anschließend wird $3 \mathrm{Min}$. zentrifugiert $(3000 \mathrm{U} / \mathrm{min})$ und das Sediment zweimal mit je $1 \mathrm{ml}$ eiskalter $0,25-n$. $\mathrm{HClO}_{4}$ gewaschen. Das Sediment wird für die weiter unten beschriebene DNS-Bestimmung verwendet. Die vereinigten Überstände werden mit 4- $n$. KOH neutralisiert (Farbumschlag) und zur besseren Abscheidung des gebildeten $\mathrm{KClO}_{4} 10$ Min. im Eisbad belassen. Nach Zentrifugierung wird der Überstand auf die Austauschersäulen dekantiert. Der Salzrückstand wird dann nochmals in $2 \mathrm{ml}$ eiskaltem Wasser aufgeschlämmt, abzentrifugiert und der Überstand ebenfalls auf die Säulen gegeben.

\section{c) Säulenchromatographie}

Für die Säulenchromatographie werden Glasrohre verwendet, die zweckmäßig eine Länge von $30 \mathrm{~cm}$ und einen Durchmesser von $1,2 \mathrm{~cm}$, am Auslauf etwas verengt und ausgezogen, besitzen und an den Boden eines 300- $\mathrm{cm}^{3}$-Erlenmeyerkolbens angeschmolzen sind (s. Abb. 1). Als Adsorbens wird

13 J. Leslie, in: E. Chargaff, J. N. Davidson, The Nucleic Acids II, 1, Academic Press, New York 1955. 
Dowex $1 \times 10,200-400$ mesh, in der Formiatform benützt. Die Harzpräparation und Säulenbereitung wird in Anlehnung an das Verfahren von Hurlbert und Mitarbb. ${ }^{11}$ durchgeführt. Hierzu wird das in

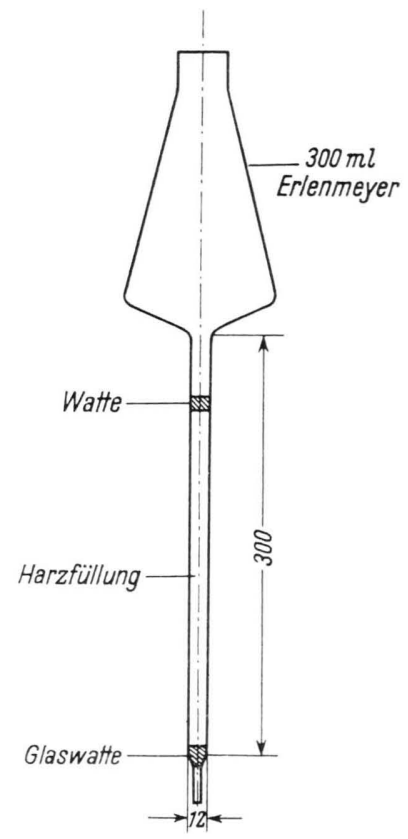

Abb. 1. Glasrohr für Säulentrennung.

der Cl-Form vorliegende Harz in Wasser (50 bis $100 \mathrm{~g} / l$ ) mehrere Male aufgeschlämmt und die kleinsten Teilchen jeweils nach dem Sedimentieren der mittleren und groben Harzpartikel abdekantiert. Dieser Schritt ist wichtig, da die Durchlaufgeschwindigkeit der Säulen sonst unnötig stark verlangsamt wird. Nachdem das Harz relativ einheitlich sedimentiert, wird es auf einer Glasfritte (z. B. Schott 29D3), oder einer Nutsche, mit 3-m. $\mathrm{NH}_{4}$-Formiat gewaschen, bis keine Cl-Ionen in der Waschflüssigkeit auftreten. Anschließend wird mit Wasser nachgewaschen und schließlich das Harz in Wasser aufgeschlämmt. Die dünnflüssige Harzsuspension wird in die unten mit wenig Glaswatte verschlossenen Säulen eingefüllt und die Säulen möglichst einheitlich gegossen. Die Höhe der Harzfüllung beträgt zweckmäßigerweise 20 Zentimeter. Kürzere Säulen verschlechtern den Trenneffekt merklich. Den oberen Abschluß der Harzfüllungen bilden jeweils Wattepfropfen. Vor Gebrauch werden die Säulen mit $200 \mathrm{~cm}^{3}$ einer 1 : 1-Mischung von $6-n$. $\mathrm{HCOOH}$ und

11 R. B. Hurlbert, H. Schmitz, H. F. Brumm ú. V. R. Potter, J. biol. Chemistry 209, 23 [1953]. 1-m. $\mathrm{NH}_{4} \mathrm{HCOO}$, dann mit $150 \mathrm{~cm}^{3}$ 88-proz. $\mathrm{HCOOH}$ und schließlich mit Wasser gewaschen, bis der Durchlauf neutral reagiert. Für Serienbestimmungen können 20-30 Säulen nebeneinander verwendet werden, wobei $1-2$ Säulen als Kontrolle dienen, die mit denselben Elutionsmitteln, aber nicht mit Substanz beschickt werden.

Auf die so vorbereiteten Säulen werden in der oben angegebenen Weise (s. b) die Überstände der Perchloratfällung gegeben. Zur Trennung der Mononucleotide werden die folgenden Elutionsmittel nacheinander verwandt:

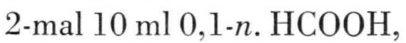

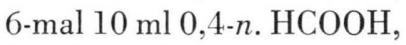
4-mal $10 \mathrm{ml}$ 2-n. $\mathrm{HCOOH}$, 8-mal $10 \mathrm{ml}$ Elutionsmittel A = 0,6-n. $\mathrm{HCOOH}+1,2-m \cdot \mathrm{NH}_{4} \mathrm{HCOO} 1: 1$, 6-mal $10 \mathrm{ml}$ Elutionsmittel $\mathrm{B}=$

$$
3-n . \mathrm{HCOOH}+\quad 3-m \cdot \mathrm{NH}_{4} \mathrm{HCOO} 1: 1 \text {. }
$$

Es werden jeweils $10 \mathrm{ml}$ auf die Säule gegeben und mit der Zugabe der nächsten Fraktion wird gewartet, bis die erste Fraktion durchgelaufen ist. Man arbeitet ohne zusätzlichen Druck, die Durchlaufgeschwindigkeit ist abhängig von der Präparation des Harzes und dem Gießen der Säulen. Sie sollte möglichst im Bereich 0,15-0,5 ml/min liegen. Die Gesamtdauer der Trennung beträgt etwa 15 bis 20 Stunden. Der Trennungsgang kann nach dem Durchlauf einer jeden Fraktion ohne weiteres für einige Zeit (z. B. nachts) unterbrochen werden, doch sollten die Säulen nicht zu lange im stark sauren Milieu belassen werden $(2-n$. HCOOH). Eine Austrocknung der Säulen ist bei kürzeren Zeiten nicht zu befürchten. Langsam laufende Säulen erzielen gute Trennungen, benötigen aber lange Versuchszeiten, zu schnell laufende Säulen ergeben unscharfe Trennungen.

Die Eluate werden jeweils in 10-ml-Fraktionen in Reagenzgläsern aufgefangen, ein automatischer Probenwechsler ist hierbei überflüssig. Nach Beendigung des Versuchs wäscht man das Harz mit etwa $200 \mathrm{~cm}^{3}$ 88-proz. $\mathrm{HCOOH}$ und anschließend mit Wasser bis zur neutralen Reaktion. Das Harz kann unmittelbar für neue Versuche verwendet werden, doch ist es zweckmäßig, zur Auflockerung des Harzes die Säulen neu zu gießen.

\section{d) Berechnung der Analysenwerte}

In den Eluaten wird die Extinktion einer Probe von jeder 10-ml-Fraktion bei $1 \mathrm{~cm}$ Schichtdicke und 
bei 260 und $280 \mathrm{~m} \mu$ gemessen. Als Vergleichswert dient jeweils die entsprechende Fraktion aus der Kontrollsäule. Die ersten beiden Eluate (0,1-n. $\mathrm{HCOOH}$ ) werden verworfen, sie enthalten absorbierende Verunreinigungen, Basen und Nucleoside. Bei den nun folgenden Fraktionen werden nur solche berücksichtigt, deren Extinktion über 0,02 liegt.

Als erstes Nucleotid wird Cytidylsäure (CMP, $\left.2^{\prime}+3^{\prime}\right)$ mit 0,4-n. HCOOH eluiert. Das Verhältnis der Extinktionen $280 \mathrm{~m} \mu / 260 \mathrm{~m} \mu$ für reines CMP beträgt 2,0. Um den Gesamtgehalt an CMP zu erhalten, werden nur solche Fraktionen berücksichtigt, deren Verhältnis 280/260 $\mathrm{m} \mu$ über 1,5 liegt. Der Gehalt an CMP wird aus der Extinktion bei $260 \mathrm{~m} \mu$ berechnet. Zweckmäßigerweise addiert man die $260 \mathrm{~m} \mu$ Extinktionen aller in Frage kommenden Fraktionen zu einer Gesamtextinktion $E_{260}$ CMP. Die Umrechnung in $\mu \mathrm{Mol}$ bzw. in mg erfolgt dann mit den weiter unten angegebenen Faktoren.

Bei der folgenden Elution mit 2-n. HCOOH können zunächst noch kleine Mengen von CMP erscheinen, was sich in dem 280/260 m $\mu$-Verhältnis bemerkbar macht. Dann tritt Adenylsäure $\left(2^{\prime}+3^{\prime}\right.$, AMP) auf, deren $280 / 260 \mathrm{~m} \mu$-Verhältnis bei 0,22 liegt. Bei der Addition der Gesamtextinktion der AMP bei $260 \mathrm{~m} \mu$ werden nur solche Fraktionen berücksichtigt, deren Verhältnis $280 / 260 \mathrm{~m} \mu$ unter 0,35 liegt.

Mit dem nun folgenden Elutionsmittel A erhält man zunächst die Uridylsäure $\left(2^{\prime}+3^{\prime}\right.$, UMP) und danach die ersten Fraktionen der Guanylsäure $\left(2^{\prime}+3^{\prime}\right.$, GMP). Die $280 / 260 \mathrm{~m} \mu$-Verhältnisse betragen 0,33 bzw. 0,68. Es werden die Fraktionen mit einem Verhältnis $280 / 260 \mathrm{~m} \mu$ unter 0,4 zur Gesamt-UMP-Extinktion bei $260 \mathrm{~m} \mu$ addiert, während die Fraktionen mit 280/260 $\mathrm{m} \mu$ zwischen 0,41 und 0,7 als GMP addiert werden. Den größten Teil des GMP erhält man mit dem Elutionsmittel B.

Zur Berechnung des Gehalts an den einzelnen Mononucleotiden bzw. der Gesamt-RNA benützt man die molaren Extinktionskoeffizienten in $\mu \mathrm{Mol} / 10 \mathrm{~cm}^{3}$ und Korrekturfaktoren für die Zerstörung der Nucleotide. Für die hier beschriebenen Bedingungen ergeben sich die folgenden Formeln:

$$
\begin{aligned}
& \mu \mathrm{Mol} \mathrm{CMP}=E_{260} \mathrm{CMP} \cdot 1,86 \\
& \mu \mathrm{Mol} \mathrm{AMP}=E_{260} \mathrm{AMP} \cdot 0,78 \\
& \mu \mathrm{Mol} \mathrm{GMP}=E_{260} \mathrm{GMP} \cdot 0,87 \\
& \mu \mathrm{Mol} \mathrm{UMP}=\left(E_{260} \mathrm{UMP}-0,22 E_{260} \mathrm{CMP}\right) \cdot 1,045 .
\end{aligned}
$$

Für die Berechnung der Nucleotidreste in der RNS (Mol.-Gew. minus $\mathrm{H}_{2} \mathrm{O}$ ) in mg bzw. für die Gesamt- menge der RNS in mg ergeben sich folgende Formeln:

$$
\begin{aligned}
& \mathrm{mg} \text { CMP-Rest }=E_{260} \mathrm{CMP} \cdot 0,568 \\
& \mathrm{mg} \text { AMP-Rest }=E_{260} \mathrm{AMP} \cdot 0,257 \\
& \mathrm{mg} \text { GMP-Rest }=E_{260} \mathrm{GMP} \cdot 0,300 \\
& \mathrm{mg} \mathrm{UMP}-\text { Rest }=\left(E_{260} \mathrm{UMP}-0,22 E_{260} \mathrm{CMP}\right) \cdot 0,320
\end{aligned}
$$

$\mathrm{mg}$ RNS $=$ Summe.

\section{e) DNS-Bestimmung}

Die DNS, als Bezugssystem für die RNS notwendig, wurde kolorimetrisch nach der Methode von Burton ${ }^{12}$ bestimmt. Von $100 \mathrm{mg}$ Trockenpulver (WTS) ausgehend, wird nach Beendigung der Hydrolyse mit Alkali und nach dem Ansäuern des Extrakts mit 70-proz. $\mathrm{HClO}_{4}$ das Sediment abzentrifugiert und gewaschen. Dieses gewaschene Sediment wird mit $0,5-n . \mathrm{HClO}_{4}$, zunächst mit $3 \mathrm{~cm}^{3}$, danach mit $2 \mathrm{~cm}^{3}$, jeweils bei $70{ }^{\circ} \mathrm{C}$ für $15 \mathrm{Min}$. extrahiert. $1-2 \mathrm{ml}$ davon werden mit 2 Volumina Burton Reagenz versetzt und $16-20 \mathrm{Stdn}$. bei $30{ }^{\circ} \mathrm{C}$ im Thermostaten belassen. Gemessen wird gegen Kontrolle $\left(0,5-n . \quad \mathrm{HClO}_{4}\right)$ und Standard-DNS bei $600 \mathrm{~m} \mu$. An Hand der mitgeführten Standard-DNS und einer Eichkurve kann die gefundene Extinktion $E_{600 \mathrm{~m}_{\mu}}$ sofort in $\gamma$ DNS umgerechnet werden.

\section{f) Papierchromatographische Untersuchung der Fraktionen}

Bei der Ausarbeitung der Methode wurde jede Fraktion papierchromatographisch auf ihre Zusammensetzung geprüft. Bei schwierigen Trennungen empfiehlt es sich, diese Kontrollen zumindest bei den zweifelhaften Fraktionen ebenfalls durchzuführen. Hierzu werden die Fraktionen nach Messungen der Extinktion im Rotationsverdampfer eingeengt und die Nucleotide papierchromatographisch in einem System getrennt, das aus 65 Volumenteilen Isopropanol, 25 Volumenteilen konz. HCl und 10 Volumenteilen Wasser besteht. Bei absteigender Chromatographie beträgt die Laufzeit etwa $40 \mathrm{Stdn}$., bei Benützung der Papiere Schleicher-Schüll $2043 \mathrm{~b}$ Mg 1 und Macherey und Nagel Nr. 212. Die Kennzeichnung der Nucleotidflecken erfolgt in der üblichen Weise unter einer Quarzlampe mit UG-5-Filter (Schott \& Genossen).

Als Bezugssubstanzen wurden Nucleotide, Nucleoside und die entsprechenden Basen der California

${ }^{12}$ K. Burton, Biochem. J. 62, 315 [1956]. 
Corporation of Biochemical Research (Reinheitsgrad A) benützt. Es ist jedoch darauf zu achten, daß diese Substanzen mehrere Prozent Verunreinigungen enthalten können und daher teilweise nochmals chromatographisch gereinigt werden müssen.

Besprechung des Analysengangs

Der Gesamttrennungsgang ist in dem Schema nochmals zusammengefaßt. Die günstigsten Arbeitsbedingungen ergeben sich im Bereich 0,5-3 $\mu \mathrm{Mol}$ je Mononucleotid, da bei höheren Konzentrationen sich die Banden bei der Säulenchromatographie überschneiden und bei niedrigeren Konzentrationen die Extinktionsmessungen unsicher werden. Die Durchführung des gesamten Trennungsgangs erfordert etwa 3 Tage, wobei allerdings ohne weiteres 20 - 30 Analysen gleichzeitig durchgeführt werden können. Eine Vereinfachung ist ohne Einbuße an Genauigkeit nicht möglich.

Bei der Vorbereitung des Pflanzenmaterials lehnten wir uns an die in der Literatur angegebenen Verfahren $^{1,13}$ an. Wichtig ist vor allem, daß ein enzymatischer oder chemischer Abbau der Nucleinsäuren vermieden wird, gleichzeitig aber alle säurelöslichen Phosphate, Phospholipide und stark absorbierenden Substanzen, wie Chlorophyll und andere Farbstoffe, entfernt werden. Die Extraktion der säurelöslichen Phosphate muß unbedingt in der Kälte und sehr schnell durchgeführt werden, da andernfalls die Hydrolyse der RNS und eine Abspaltung der Purinreste an der DNS eintreten können. Unsere Versuche ergaben, daß das anschließende Nachwaschen mit eiskaltem Wasser nicht zu Verlusten an RNS führt. Es ist aber streng darauf zu achten, daß die $\mathrm{HClO}_{4}$ vollständig aus dem Gewebe-Rückstand entfernt wird, da sonst beim nachfolgenden Erhitzen mit organischen Lösungsmitteln eine Abspaltung der Purine erfolgt, die die spätere Säulenchromatographie stark stört.

$$
\text { Alkalische Hydrolyse }
$$

Die alkalische Hydrolyse ist der kritische Punkt des Trennungsgangs, und die von den verschiedenen Autoren angegebenen Bedingungen unterscheiden sich stark. Deshalb wurden die Bedingungen hierfür besonders sorgfältig geprüft. In den einzelnen Versuchsreihen wurde die Alkalikonzentration zwischen $0,2-2-n$. KOH und die Temperatur zwischen 20 und $50{ }^{\circ} \mathrm{C}$ variiert. Das Problem besteht darin, eine vollständige Hydrolyse der in dem Pflan- zenmaterial vorhandenen RNS zu Mononucleotiden unter möglichst schonenden Bedingungen zu erreichen, ohne daß die DNS hierbei säurelöslich wird.

Wir prüften zunächst die Zersetzungsgeschwindigkeit jedes der vier Mononucleotide bei $37{ }^{\circ} \mathrm{C}$ und bei $50{ }^{\circ} \mathrm{C}$ mit 1-n. $\mathrm{KOH}$, wobei der Verlust an Mononucleotiden durch Säulenchromatographie, aber auch papierchromatographisch ermittelt wurde. Abb. 2 zeigt die bei $37{ }^{\circ} \mathrm{C}$ erhaltenen Ergebnisse.

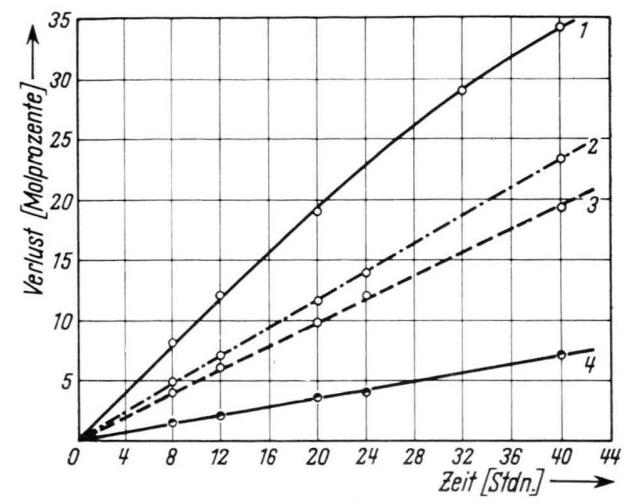

Abb. 2. Einwirkung von 1-n. KOH auf $10^{-3}-m$. Mononucleotide bei $37^{\circ} \mathrm{C}: 1=$ CMP-Totalverlust, $2=$ CMP-Verlust durch Desaminierung zu UMP, $3=$ AMP-Verlust, $4=$ UMPVerlust.

Erwartungsgemäß ist die Zersetzung bei CMP am stärksten. Überraschenderweise werden jedoch AMP und in geringerem Maße UMP, die bisher als stabil galten, ebenfalls zersetzt. Nur bei GMP wurde unter den angegebenen Bedingungen kein Verlust beobachtet. Kurve 2 zeigt, daß nur ein Teil des verlorengegangenen CMP zu UMP desaminiert wird, während der übrige Teil auf eine unbekannte Weise verändert wird. Wegen des Verlusts der Extinktion bei $260 \mathrm{~m} \mu$ ist eine Aufspaltung des Pyrimidinrestes wahrscheinlich. Die Ursache für die Zerstörung der AMP und UMP ist ebenfalls unbekannt. Bei der Suche nach Zersetzungsprodukten der AMP konnten nach sehr langen Hydrolysezeiten geringe Spuren einer Substanz gefunden werden, die sich papierchromatographisch und uv-spektrometrisch wie Inosinsäure verhält. Nur durch Desaminierung läßt sich also der Verlust an AMP kaum erklären. Da bei allen AMP-Fraktionen ein Extinktionsverlust im gesamten Meßbereich von 205-305 m $\mu$ auftritt, wird auf eine teilweise Zersetzung des Purinskeletts geschlossen. Die Zersetzung von CMP, AMP und UMP folgt bei $37^{\circ} \mathrm{C}$ annähernd einer Reaktion erster Ordnung, so daß es einfach ist, aus der Abb. 2 die 
Verluste auch für andere Hydrolysezeiten zu entnehmen.

Bei $50{ }^{\circ} \mathrm{C}$ (Abb. 3) steigt die Zersetzungsgeschwindigkeit bei allen Nucleotiden an, nur GMP bleibt auch hier stabil. Überraschenderweise ist die Zersetzung jedoch bei AMP ungewöhnlich hoch und

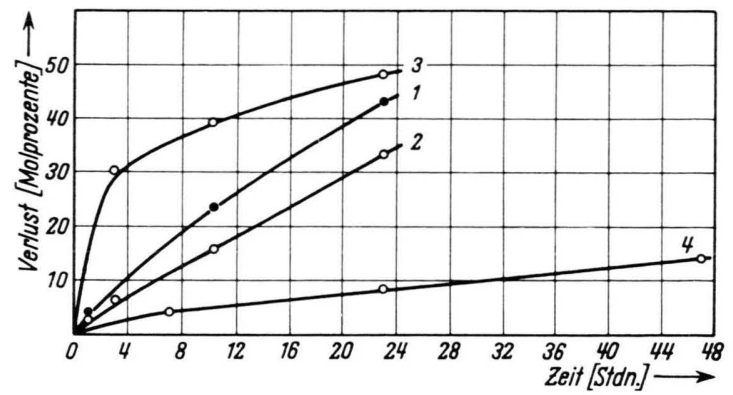

Abb. 3. Einwirkung von 1-n. KOH auf $10^{-3}-m$. Mononucleotide bei $50{ }^{\circ} \mathrm{C}: 1=$ CMP-Totalverlust, $2=$ CMP-Verlust durch Desaminierung, 3 = AMP-Verlust, $4=$ UMP-Verlust.

läßt sich nicht mehr durch eine Reaktion erster Ordnung beschreiben. Wegen dieser unübersichtlichen Verhältnisse ist die Anwendung höherer Hydrolysentemperaturen nicht zu empfehlen.

Anschließend wurde untersucht, mit welcher Geschwindigkeit die Mononucleotide aus den Ribonucleinsäuren in Freiheit gesetzt werden. Hierzu wurden sowohl mit reiner Hefe-RNS als auch mit dem RNS-haltigen Blattmaterial von Nicotiana tabacum (Samsun) und Epilobium parviflorum $\mathrm{Hy}$ drolyse-Versuche unternommen. $\mathrm{Zu}$ verschiedenen Versuchszeiten wurden Proben entnommen und die jeweils vorhandenen Mengen an Mononucleotiden durch Säulenchromatographie getrennt. Es ergab sich in allen Fällen ein gleichartiger Verlauf, so daß wir uns auf die Wiedergabe je eines Versuchsbeispiels für Hefe-RNS und Blattmaterial von Tabak beschränken können. Der Übersichtlichkeit halber ist der Verlauf für jedes Mononucleotid in den Abb. $4 \mathrm{a}-4 \mathrm{~d}$ wiedergegeben. In allen Fällen steigt

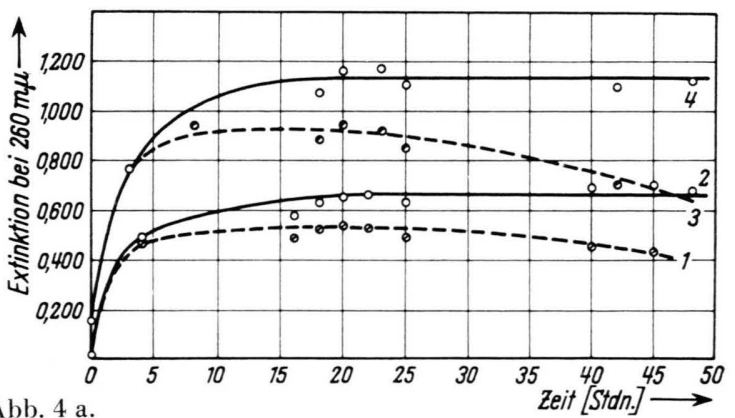

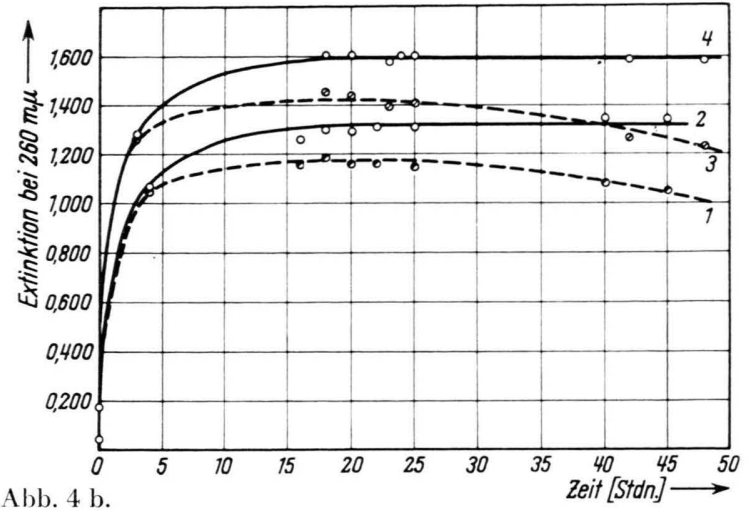
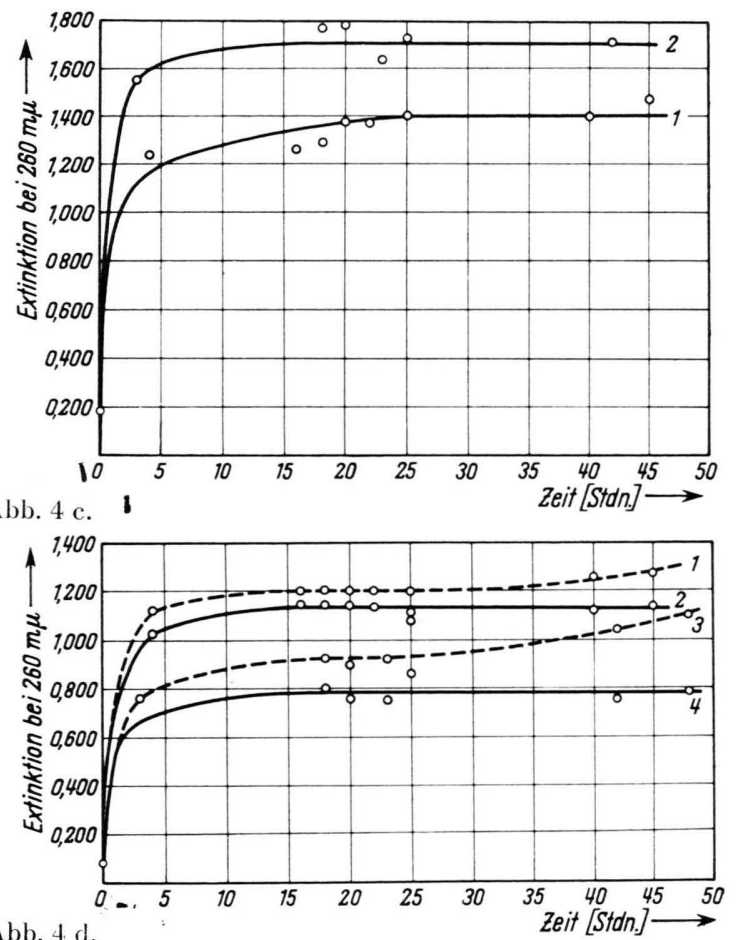

Abb. 4 d.

Abb. 4. Hydrolyse von Hefe-RNS $\left(2 \mathrm{mg} / 10 \mathrm{~cm}^{3}\right)$ und Tabakblattmaterial $\left(100 \mathrm{mg}\right.$ Trockensubstanz $\left./ 10 \mathrm{~cm}^{3}\right)$ in $1-n . \mathrm{KOH}$ bei $37^{\circ} \mathrm{C}$ : a) CMP-Konzentration im Hydrolysat $1=\mathrm{CMP}$ aus Hefe gemessen, $2=$ CMP aus Hefe berechnet (gemessen + Verlust), $3=$ CMP aus Tabak gemessen, $4=\mathrm{CMP}$ aus Tabak berechnet (gemessen+Verlust). b) AMP-Konzentration im Hydrolysat $1=\mathrm{AMP}$ aus Hefe gemessen, $2=$ AMP aus Hefe berechnet (gemessen + Verlust), $3=\mathrm{AMP}$ aus Tabak gemessen, $4=$ AMP aus Tabak berechnet (gemessen + Verlust). c) GMP-Konzentration im Hydrolysat $1=$ GMP aus Hefe gemessen, $2=$ GMP aus Tabak gemessen. d) UMP-Konzentration im Hydrolysat $1=$ UMP aus Hefe gemessen, 2 = UMP aus Hefe berechnet (gemessen + Verlust minus UMP aus CMP), $3=$ UMP aus Tabak gemessen, $4=$ UMP aus Tabak berechnet (gemessen + Verlust minus UMP aus CMP).

die Menge an Nucleotiden in den ersten $3-4$ Stdn. stark an. Während dieser Zeit sind chromatogra- 
phisch auch Oligonucleotide nachweisbar. Bei AMP (Abb. 4 b) und CMP (Abb. 4 a) erreicht die Kurve ein flaches Maximum, um dann infolge Zersetzung der Mononucleotide wieder abzusinken. Addiert man zu der experimentell gefundenen Menge von CMP und AMP jeweils ihre Verluste (entsprechend Abb. 2), so erhält man ab 22 Stdn. einen konstanten Wert, d.h. daß nunmehr kein weiteres Mononucleotid mehr freigesetzt wird und die Hydrolyse beendet ist. Bei GMP (Abb. 4c) ergibt sich ohne Korrektur der Endpunkt der Hydrolyse ebenfalls nach 22 Stunden. Die Menge an UMP (Abb. $4 \mathrm{~d}$ ) im Hydrolysat steigt nach $22 \mathrm{Stdn}$. noch weiter an, da das aus CMP neu gebildete UMP den Verlust übertrifft. Addiert man einerseits den Verlust und subtrahiert andererseits das aus CMP entstandene UMP unter Benützung der Kurven 2 und 4 der Abb. 2, so erhält man ebenfalls nach $22 \mathrm{Stdn}$. einen konstanten Wert.

Diese Versuchsserie zeigt also, daß die an den reinen Mononucleotiden gemessene Zerstörung in gleicher Weise auch bei der Hydrolyse von RNS in Gegenwart größerer Mengen von Proteinen und anderen Verunreinigungen auftritt. Um die hierfür notwendigen Korrekturen auf ein Minimum zu beschränken, sollte man daher die Hydrolyse nicht über $22 \mathrm{Stdn}$. hinaus durchführen. Bei niedrigeren $\mathrm{KOH}-$ Konzentrationen oder bei tieferen Temperaturen werden die Mononucleotide langsamer zersetzt, allerdings ergeben sich sehr lange Hydrolysenzeiten (z. B. bei 0,2-n. KOH mehr als $40 \mathrm{Stdn}$.). Diese langen Versuchszeiten sind für den Routine-Betrieb sehr unbequem. Eine Erhöhung der KOH-Konzentration etwa auf $2-n$. oder eine Steigerung der Temperatur bis $50{ }^{\circ} \mathrm{C}$ setzt zwar die Hydrolysendauer herab, aber die Zersetzung der Mononucleotide wird dann unübersichtlich.

In der Literatur ${ }^{1}$ wird weiterhin darauf hingewiesen, daß bei höherer Temperatur die DNS säurelöslich wird. Wir überzeugten uns durch entsprechende Kontrollversuche, daß dies unter den von uns angegebenen Bedingungen bei $37^{\circ} \mathrm{C}$ nicht der Fall ist. Zur Kontrolle, ob mit unseren Hydrolyse-Bedingungen wirklich die Gesamt-RNS erfaßt wird, extrahierten wir nach der Säurefällung den Rückstand der alkalischen Hydrolyse mit 0,5-n. $\mathrm{HClO}_{4}$ (2-mal je 15 Min.) bei $70^{\circ} \mathrm{C}$. Die kolorimetrische Prüfung mit Orcin ${ }^{14}$ fiel jedoch negativ aus.

\section{$\mathrm{S} \ddot{\mathrm{u} u l e n t r e n n u n g}$}

Die Trennung von Mononucleotiden mit Hilfe von Austauscherharzen hat sich vielfach bewährt, doch mußte die Methode den besonderen Bedingungen der Pflanzenanalyse angepaßt werden. Als Austauscherharz benützten wir Dowex $1 \times 10$ und prüften die Elutionsmittel Ameisensäure und Formiat in verschiedenen Konzentrationen. Mit Ammoniumformiat war die Trennung in die beiden Gruppen der Pyrimidin- und Purin-Nucleotide gut, innerhalb dieser Gruppen aber unbefriedigend. Mit reiner Ameisensäure verschiedener Konzentrationen war die Trennung aller 4 Nucleotide voneinander gut, wie dies aus Abb. 5 hervorgeht, doch waren die Werte für die einzelnen Nucleotide schwer reproduzierbar. Bei diesem Verfahren muß bis zu 6- $n$. $\mathrm{HCOOH}$ benützt werden, und bei diesen hohen Konzentrationen macht sich deren Eigenextinktion bei $260 \mathrm{~m} \mu$ störend bemerkbar. Außerdem kann es durch die hohe Säurekonzentration schon zur Abspaltung von Purinen kommen.

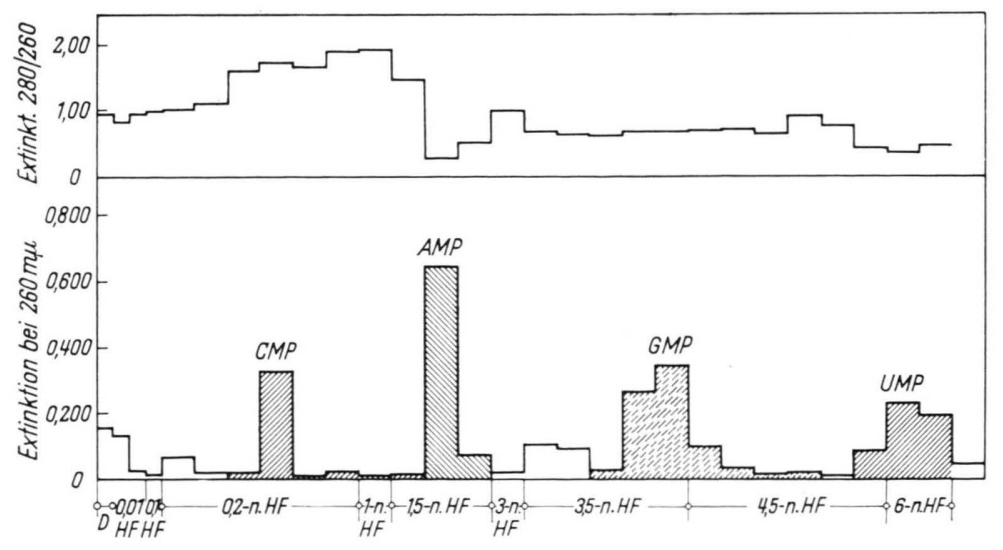

14 G. Ceriotti, J. biol. Chemistry 214, 59 [1955].
Abb. 5. Trennung eines Hydrolysats aus Tabakblättern an Dowex $1 \times 10 \mathrm{mit}$ steigenden Konzentrationen von Ameisensäure (50 mg WTS/10 ml l-n. KOH, 22 Stdn. bei $37^{\circ} \mathrm{C}$ ). 
Abb. 6. Trennung eines Hydrolysats aus Tabakblättern an Dowex $1 \times 10$ mit Mischungen aus $\mathrm{HCOOH}$ und $\mathrm{NH}_{4} \mathrm{HCOO}$ wie im Analysengang vorgeschlagen $(50 \mathrm{mg}$ WTS $/ 10 \mathrm{ml}, 1-n$. $\mathrm{KOH}, 22 \mathrm{Stdn}$. bei $37^{\circ} \mathrm{C}$ ), schraffierte Flächen: Mononucleotide, weiße Flächen: absorbierende Begleitstoffe.

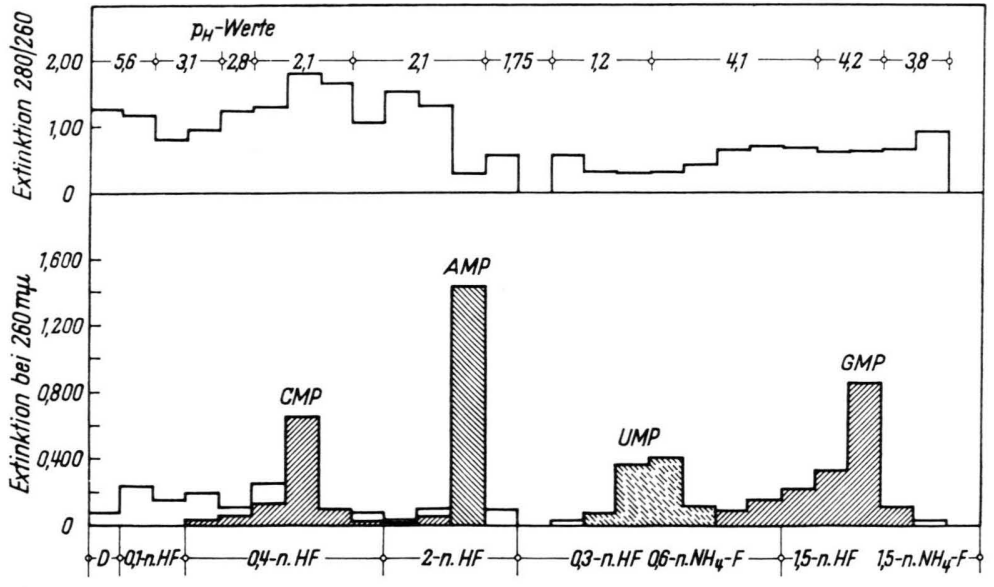

nicht allzu groß ist. Falls bei einer Analyse höhere $E_{260}$-Werte in diesen Fraktionen auftreten, deren Extinktionsverhältnis nicht den angegebenen Werten entspricht, ist eine papierchromatographische Nachprüfung zu empfehlen *. Um größere Genauigkeit zu erreichen, kann bei diesen Vorfraktionen aus dem Verhältnis $E_{280} / E_{260}$ die Menge an Guanin und CMP berechnet und bei dem Gesamtwert berücksichtigt werden.

\section{Fehlerdiskussion}

Die für die Berechnung der Analysendaten zugrunde gelegten molaren Extinktionskoeffizienten, ferner die Verhältnisse der Extinktionen 280/260 m $\mu$ sind aus Tab. 1 zu entnehmen. Ferner enthält die Tabelle die Korrekturfaktoren, die sich infolge Verlusten bzw. Transformation von CMP zu UMP bei 22 -stdg. Hydrolyse ergeben.

Mit der hier beschriebenen Methode wurden annähernd 100 Analysen, hauptsächlich an Tabak und an Epilobium durchgeführt. Über das Ergebnis dieser Untersuchungen wird in anderem Zusammenhang berichtet werden. Um die Genauigkeit der Methode zu prüfen, wurden einzelne Bestimmungen 6-8-mal wiederholt. Bei der statistischen Auswergung der Fraktionen mit 280/260 m $\mu<1,5$ entsteht,

\begin{tabular}{|c|c|c|c|c|c|}
\hline Nucleotid & $p \mathrm{H}$ & $E_{280 \mathrm{~m} \mu} / E_{260 \mathrm{~m} \mu} 15$ & $\begin{array}{c}E \text { je } \mu \mathrm{Mol} / 10 \mathrm{ml} \\
\text { bei } 260 \mathrm{~m} \mu\end{array}$ & $\begin{array}{c}\text { Verlust } V \\
\text { in Mol-Proz. }\end{array}$ & $100 /(100-V) E$ \\
\hline GMP & $3-7$ & 0,68 & 1,16 & 0 & 0,87 \\
UMP & $1-6$ & 0,33 & 0,99 & 3,3 & 1,045 \\
AMP & $1-2$ & 0,22 & 1,43 & 11,1 & 0,78 \\
CMP1 & $1-2$ & 2,0 & 0,67 & 20,4 & 1,86 \\
CMP2 & $1-2$ & 2,0 & 0,67 & 11,8 & \\
\hline
\end{tabular}

Tab. 1. Extinktionen der Mononucleotide und Verluste bei 22 Stdn. Hydrolyse in 1-n. KOH bei $37^{\circ} \mathrm{C} .1^{1}$ Totalverlust. 2 Mol.-Prozent CMP, die in UMP umgewandelt werden. Die Menge an UMP aus CMP ergibt sich zu $1,86 \cdot 0,118 \cdot E_{\mathrm{CMP}}$.

15 K. Burton, Data for Biochem. Research, The Clarendon Press, Oxford 1959.
* So konnten z. B. bei Epilobium bis zu 5\% anormale Nucleotide, bezogen auf die Gesamt-RNS, nachgewiesen werden. 


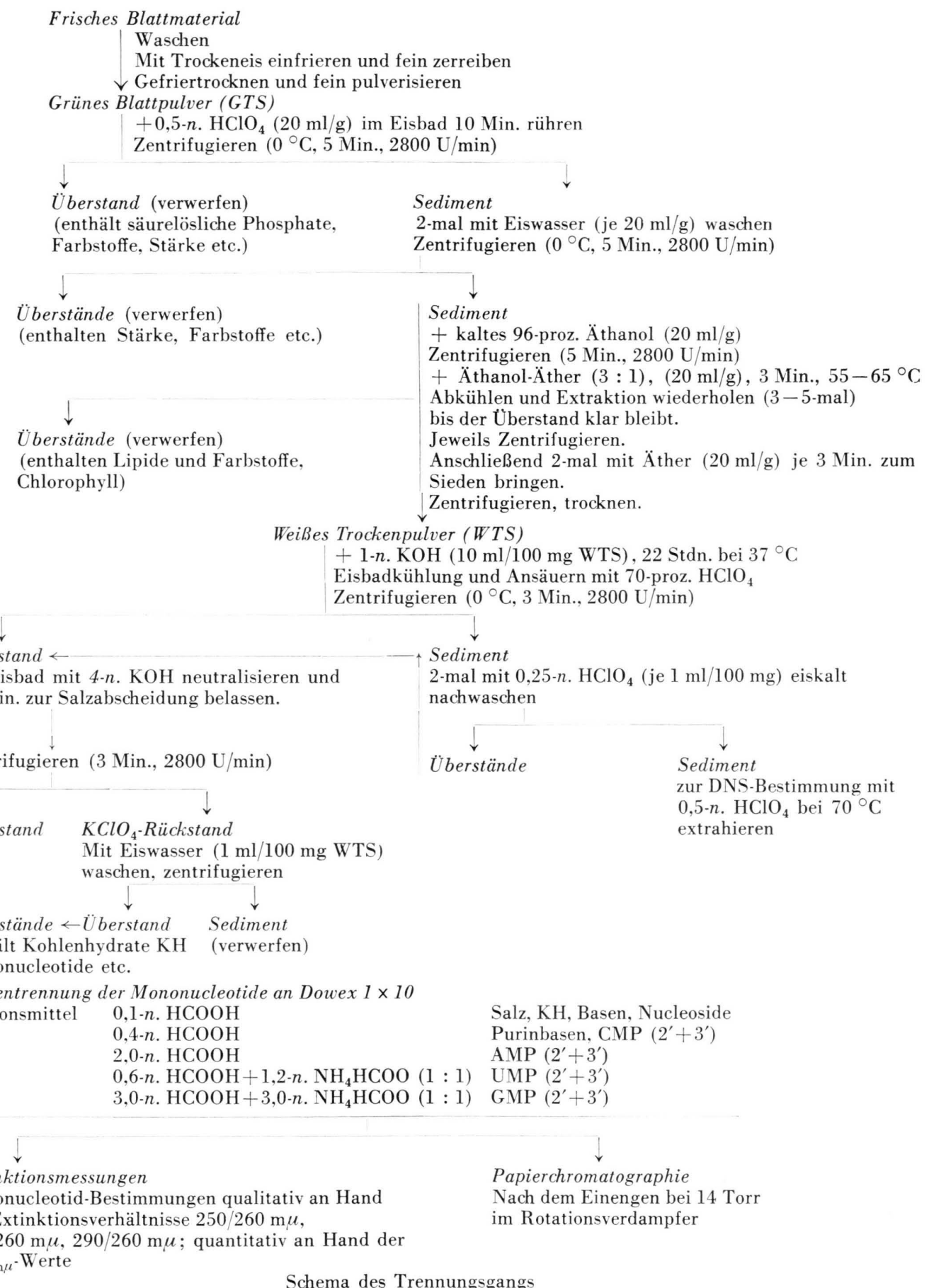

tung dieses Materials ergab sich für die Einzelbestimmung ein mittlerer Fehler von \pm 5 Prozent. In Anbetracht der zahlreichen Operationen, die durchzuführen sind, darf das als befriedigend betrachtet werden.
Herm Prof. Dr. G. Schramm danken wir für die Unterstützung der Arbeit und für wertvolle Diskussion, Herrn W. Vö̈sch für sorgfältige technische Mitarbeit.

Wir danken der Deutschen Forschungs. gemeinschaft und dem US Public Health S e rvice für finanzielle Unterstützung. 